Kinetic space-time prisms

Peer-reviewed author version

KUIJPERS, Bart; OTHMAN, Walied \& Miller, Harvey J. (2011) Kinetic space-time prisms. In: Cruz, Isabel F.; Agrawal, Divyakant; Jensen, Christian S.; Ofek, Eyal; Tanin, Egemen (Ed.). Proceedings of the 19th ACM SIGSPATIAL International Conference on Advances in Geographic Information Systems, p. 162-170.

DOI: $10.1145 / 2093973.2093996$

Handle: http://hdl.handle.net/1942/13255 


\section{Kinetic space-time prisms}

\author{
Bart Kuijpers \\ Hasselt University \\ Database and Theoretical \\ Computer Sciences Research \\ Group \\ Bart.Kuijpers@ \\ uhasselt.be
}

\author{
Harvey J. Miller \\ University of Utah \\ Department of Geography \\ harvey.miller@ \\ geog.utah.edu
}

\author{
Walied Othman \\ University of Muenster \\ Institute For Geolnformatics \\ (ifgi) \\ walied.othman@ \\ gmail.com
}

\begin{abstract}
The space-time path and prism demarcate the estimated and potential locations (respectively) of a moving object with respect to time. The path is typically formed through linear interpolation between sampled locations of a moving object, while the prism is the envelope of all possible paths between two locations given the maximum speed of travel. The classic path and prism, however, are not physically realistic since they imply the ability of the object to make instantaneous changes in direction and speed without acceleration and deceleration. This is not acceptable in applications where kinetics is vital for scientific understanding such as animal ecology, vehicles moving through media such as ships through water and planes through air, human-powered movement such as bicycling and walking and environmental applications of transportation such as energy consumption and emissions modeling. In this paper we demonstrate how imposing an upper bound on acceleration, as well as information such as the initial speed and heading, affects the geometry of the space-time prism. We discuss how to calculate kinetic paths and prisms in one-dimensional and two dimensional space, and provide examples comparing the kinetic prisms and classical prisms.
\end{abstract}

\section{INTRODUCTION}

The space-time path and prism are central concepts in a wide range of fields concerned with mobile objects such as vehicles, people and animals. The space-time path represents actual mobility; this is typically a polyline in twodimensional space and time constructed though linear interpolation between locations sampled by devices such as a global position system (GPS) receiver. The space-time prism represents potential mobility: this is a region in twodimensional space and time where a mobile object could be during a specific time interval, given known locations at the beginning and end of that interval (the prism anchors) and a speed limit on the object's movement. We can interpret this region as a measure of the object's accessibility to an envi- ronment [7]. The prism, however, can also represent nonsampled locations between locational fixes in the space-time path, particularly when the path is undersampled and we wish to represent the resulting error regions explicitly [18].

Although the space-time path and prism represent actual and potential movement, they are not physically realistic. The space-time path implies the ability to make instantaneous changes in direction and speed (at the sampled locations) without acceleration and deceleration. Similarly, the space-time prism implies the ability to instantly accelerate and decelerate when leaving and arriving (respectively) at anchors, as well as make directional changes instantly at its boundaries. These unrealistic geometries manifest since the path and prism consider only the object's speed limit and not limits on acceleration and deceleration that are inherent in physical movement.

Ignoring the kinetics of the space-time path and prism may be acceptable in applications such as transportation and migration where the emphasis on estimating meso and macroscale patterns from micro-level mobility data; although even here the traditional prism will overstate the region accessible to the object. There are applications, however, where physically realistic representation of an object's movement can be critical; these include animal movement [5, 6, 19, 21], vehicles moving through media such as ships through water and planes through air [9], and human-powered movement such as bicycling and walking [8, 17]. The kinetics of powered vehicles can also be important for some transportation applications such as traffic flow, resource use, emissions and safety $[2,1]$.

One way to deal with the problem of unrealistic mobility in the space-time prism is by further constraining the possible trajectories. Since a space-time prism is already defined as the envelope of all trajectories between two anchors given an upper bound on their speed, the next logical step is to constrain the possible acceleration and deceleration exhibited by these trajectories. This will ensure that a trajectory contains no instantaneous changes in direction and speed. In this paper, we will cover how imposing an upper bound on acceleration affects the geometry of the space-time prism. It turns out that this is a much richer set of shapes and that we can add or leave out additional parameters such as initial speed, initial heading or a combination of both.

In Section 2, we start with laying out the basic notions of 
trajectories and the classical space-time prism. In Section 3, we introduce the notion of kinetic paths and kinetic prisms, which are trajectories and sets of trajectories which have upper bounds on their speed and acceleration. In Section 4, we give a comprehensive list of shapes of kinetic prisms and list the effects the different parameters have on the shape. We do this both for movement in one and two dimensions.

\section{BASIC CONCEPTS}

We start with some basic definitions. All the definitions in this section are for movement in two-dimensional space, the same definitions for movement on a one-dimensional line can easily be obtained by dropping one spatial component.

Definition 1. Let $I \subseteq \mathbf{R}$ be an interval. A trajectory $T$ is the graph of a piece-wise $\operatorname{smooth}^{1}$ (with respect to $t$ ) mapping

$$
\alpha: I \subseteq \mathbf{R} \rightarrow \mathbf{R}^{2}: t \mapsto \alpha(t)=\left(\alpha_{x}(t), \alpha_{y}(t)\right),
$$

i.e., $T=\left\{\left(\alpha_{x}(t), \alpha_{y}(t), t\right) \in \mathbf{R}^{2} \times \mathbf{R} \mid t \in I\right\}$. The set $I$ is called the time domain of $T$.

From this definition, we can derive what velocity and acceleration vectors look like in space-time. The velocity vector to a trajectory $\left(\alpha_{x}(t), \alpha_{y}(t), t\right)$ is $\left(\alpha_{x}^{\prime}\left(t_{0}\right), \alpha_{y}^{\prime}\left(t_{0}\right), 1\right)$ at a time $t_{0}$, where $\alpha^{\prime}$ denotes the derivative of $\alpha$ wrt $t$. The acceleration vector to a trajectory $\left(\alpha_{x}(t), \alpha_{y}(t), t\right)$ at a time $t_{0}$ is $\left(\alpha_{x}^{\prime \prime}\left(t_{0}\right), \alpha_{y}^{\prime \prime}\left(t_{0}\right), 0\right)$, where $\alpha^{\prime \prime}$ denotes the second derivative of $\alpha$ wrt $t$. Note that the temporal component of the velocity vector is always 1 and that the temporal component of the acceleration vector is always 0, i.e., it is always parallel to the spatial plane. This means that the length of the acceleration vector at a moment in time equals the acceleration of the trajectory at that moment, this is not the case for the velocity vector.

In practice, however, we will hardly ever have a trajectory at our disposal, but rather discrete time-stamped locations of a moving object, also called a trajectory sample.

Definition 2. A trajectory sample is a finite set of timespace points $\left\{\left(x_{0}, y_{0}, t_{0}\right),\left(x_{1}, y_{1}, t_{1}\right), \ldots,\left(x_{N}, y_{N}, t_{N}\right)\right\}$, where the order on time, $t_{0}<t_{1}<\cdots<t_{N}$, induces a natural order.

A path is an interpolated space-time curve between the sample points, parametrized in time such that the curve's location at time $t_{i}$ is $\left(x_{i}, y_{i}\right)$.

Let $p=\left(x_{p}, y_{p}, t_{p}\right), q=\left(x_{q}, y_{q}, t_{q}\right) \in \mathbf{R}^{2} \times \mathbf{R}$ be two spatiotemporal points, where $t_{p}<t_{q}$. The minimal (average) speed to get from $p$ to $q$ is denoted by $v_{\min }$ and equals

$$
v_{\min }=\frac{\sqrt{\left(x_{q}-x_{p}\right)^{2}+\left(y_{q}-y_{p}\right)^{2}}}{t_{q}-t_{p}} .
$$

\footnotetext{
${ }^{1}$ Smooth is here used in the terminology of differential geometry [15], meaning differentiable or $C^{1}$.
}

What happens between these time-stamped locations is anyone's guess. Usual approaches include (linear) interpolation to connect the dots, however, these present crisp trajectories and they are merely guesses. Admittedly, linear interpolation uses the least assumptions, but the sudden changes in speed and direction at the sample points make it an unrealistic guess.

We can do something different if we have background information. If we know, for example, the speed limit of the moving object between recorded locations, we can capture all their possible trajectories (locations) in space-time prisms $[7,12,13,11,14]$.

Definition 3. Let $v_{\max } \in \mathbf{R}^{+}$and $\left(x_{p}, y_{p}, t_{p}\right),\left(x_{q}, y_{q}, t_{q}\right) \in$ $\mathbf{R}^{2} \times \mathbf{R}$, with $t_{p}<t_{q}$ be given. The space-time prism with anchors $\left(x_{p}, y_{p}, t_{p}\right)$ and $\left(x_{q}, y_{q}, t_{q}\right)$ and maximal speed $v_{\max }$, denoted by $\mathcal{P}\left(x_{p}, y_{p}, t_{p}, x_{q}, y_{q}, t_{q}, v_{\max }\right)$, is the set of points $(x, y, t) \in \mathbf{R}^{2} \times \mathbf{R}$ that satisfy the following constraints:

$$
\left\{\begin{array}{c}
t_{p} \leq t \leq t_{q} \\
\left(x-x_{p}\right)^{2}+\left(y-y_{p}\right)^{2} \leq\left(t-t_{p}\right)^{2} v_{\max }^{2} \\
\left(x-x_{q}\right)^{2}+\left(y-y_{q}\right)^{2} \leq\left(t_{q}-t\right)^{2} v_{\max }^{2}
\end{array}\right.
$$

The inequalities in Definition 3 express that a point inside the prism cannot be farther away from the first anchor than the speed limit times the elapsed time, and that it has to be closer to the second anchor than the speed limit times the remaining time. This means there exists a path through that point connecting the anchors that is less than the speed limit times the time interval between the two anchors.

Figure 1 visualizes Definition 3. On the left we have a cone pointing downward, which is expressed by the second inequality in Definition 3, and a cone pointing upward, which is expressed by the third inequality in Definition 3. This is a system of inequalities, so the point has to be in the intersection of those two cones, and this is depicted in Figure 1 on the right.

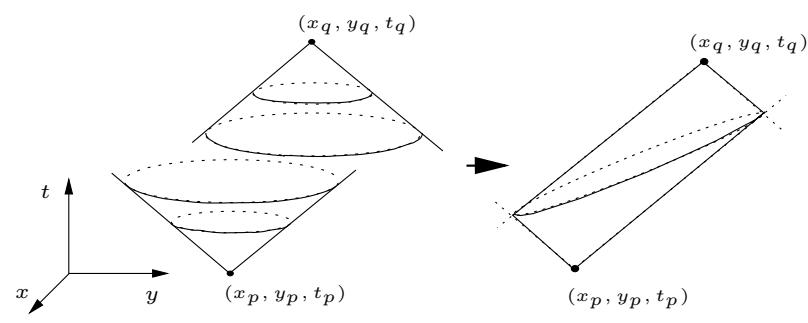

Figure 1: A space-time prism

Note that when the spatial distance between the anchors equals the speed limit times the time difference between the anchors, the space-time prism degenerates to a line connecting the anchors. Otherwise, the space-time prism captures all trajectories between two anchors which obey the speed limit.

We omitted the inclusion of stationary activity time [14] in Definition 3, and intend to include this in a more extended version of this paper. 


\section{KINETIC PATHS \& PRISMS}

Trajectories on the boundary of a space-time prism move at the speed limit and change direction instantaneously on the rim of the space-time prism, where the two cones meet. This instantaneous change in direction is unrealistic, and trajectories that approximate this behavior have an acceleration that tends to infinity as the object approaches this point of direction change.

We counter this by imposing an upper bound on the moving object's acceleration, similar to, and in addition to, the upper bound on its speed. In the case of space-time prisms, this is relatively easy to translate into equations since spatiotemporal points inside a space-time prism are characterized by only one condition on their distance to the anchors. This is not the (immediate) case when there is also an upper bound in the moving object's acceleration.

Definition 4. Let $\left(x_{p}, y_{p}, t_{p}\right),\left(x_{q}, y_{q}, t_{q}\right) \in \mathbf{R}^{2} \times \mathbf{R}$ be two anchors, where $t_{p}<t_{q}$. Let $v_{\max }, a_{\max } \in \mathbf{R}^{+}$be an upper bound on speed and acceleration respectively. A kinetic path is the graph of a $C^{2}$ (with respect to $t$ ) mapping

$$
\alpha:\left[t_{p}, t_{q}\right] \rightarrow \mathbf{R}^{2}: t \mapsto \alpha(t)=\left(\alpha_{x}(t), \alpha_{y}(t)\right),
$$

such that for all moments $t$ in the open interval between $t_{p}$ and $t_{q}$ we have that

$$
\left\|\left(\alpha_{x}^{\prime}(t), \alpha_{y}^{\prime}(t)\right)\right\| \leq v_{\max } \text { and }\left\|\left(\alpha_{x}^{\prime \prime}(t), \alpha_{y}^{\prime \prime}(t)\right)\right\| \leq a_{\max },
$$

where $\|\cdot\|$ is the Euclidean norm. In addition, each anchor may be attributed with an initial speed $v_{p} \in \mathbf{R}^{+}$and initial heading, denoted by $\left(v_{p} \cos \left(\theta_{p}\right), v_{p} \sin \left(\theta_{p}\right), 1\right)$ or just an initial speed $v_{p}$ for the anchor $\left(x_{p}, y_{p}, t_{p}\right)$.

Moreover, if an initial speed $v_{p} \in \mathbf{R}^{+}$and initial heading $\left(v_{p} \cos \left(\theta_{p}\right), v_{p} \sin \left(\theta_{p}\right), 1\right)$ are defined, then $\alpha$ has to satisfy $\alpha_{x}^{\prime}\left(t_{p}\right)=v_{p} \cos \left(\theta_{p}\right)$ and $\alpha_{y}^{\prime}\left(t_{p}\right)=v_{p} \sin \left(\theta_{p}\right)$. If only an initial speed $v_{p} \in \mathbf{R}^{+}$is defined, then $\alpha$ has to satisfy $\left\|\left(\alpha_{x}^{\prime}\left(t_{p}\right), \alpha_{y}^{\prime}\left(t_{p}\right)\right)\right\|=v_{p}$. The same constraints hold when $p$ is replaced by $q$.

We have to take care when we extend this to a trajectory sample of more than two points, and make sure that transitioning from one prism to another is always done in a $C^{1}$ fashion. Moreover, the range of a heading vector is limited by the previous sample point and its heading vector.

Next we define a kinetic prism, which, much like a spacetime prism, bounds a subset of space-time between two anchors of spatio-temporal points on kinetic paths from one anchor to the other, given upper bounds on the speed and acceleration of those paths, and with or without an initial speed and initial heading or just initial speed at the anchors.

Definition 5. Let $\left(x_{p}, y_{p}, t_{p}\right),\left(x_{q}, y_{q}, t_{q}\right) \in \mathbf{R}^{2} \times \mathbf{R}$ be two anchors, where $t_{p}<t_{q}$. Let $v_{\max }, a_{\max } \in \mathbf{R}^{+}$be an upper bound on speed and acceleration respectively. A kinetic prism is the set of all spatio-temporal points on a kinetic path from the first anchor to the second.

When an anchor is attributed with an initial speed $v_{p} \in \mathbf{R}^{+}$ and initial heading, denoted by $\left(v_{p} \cos \left(\theta_{p}\right), v_{p} \sin \left(\theta_{p}\right), 1\right)$ or just an initial speed $v_{p}$ for the anchor $\left(x_{p}, y_{p}, t_{p}\right)$, then the aforementioned kinetic paths have to satisfy these additional constraints as described in Definition 4.

This definition is unfortunately descriptive and quantifies over kinetic paths. In the following sections we will lay out the diversity that kinetic prisms offer, due to combining different initial conditions. We also provide Mathematica implementations of the algorithms we present [16]. We will also give a description of how to construct analytical characterizations of the points that can be reached via a kinetic path, however, the characterizations are too complex and including these huge expressions here would not add insight.

\section{COMPUTING KINETIC PRISMS}

In the following we will provide algorithms for the computation of the boundary of the kinetic prisms with all possible combinations of initial speeds and initial headings. Each of these have distinct topological properties and will be explored separately.

\subsection{No initial speed and initial heading}

The lack of an initial speed and heading means that from the first anchor, any heading and any speed between zero and the maximal speed is allowed. We will show that trajectories cannot achieve this upper bound on their speed if there is not enough time to change direction towards the second anchor.

Next, we will separately examine the one-dimensional and two-dimensional case. We will show how to solve the twodimensional case as an extension of the one-dimensional case.

\subsubsection{Movement on a one-dimensional line}

We can easily obtain the definitions for the one-dimensional case by ignoring the $y$-component in all the previous definitions. Figure 2 shows a standard space-time prism for a moving object between two anchors.



Figure 2: A one-dimensional space-time prism.

Since we do not constrain the object's initial speed, the prism's shape at the anchors do not need special consideration. The other corners of the prism, however, represent instant direction changes on a trajectory of an object that travels at the maximal allowed speed. It is precisely those 
trajectories that are impossible to physically realize if we impose an upper bound $a_{\max }$ on the object's acceleration.

The most general equation for an object moving with constant acceleration $a$ and a time-independent initial speed $v_{0}$ at a time $t_{0}$ and location $x_{0}$ is

$$
x=x_{0} \pm v_{0} t \pm \frac{a t^{2}}{2},
$$

so the change of direction for an object moving at maximal speed and maximal acceleration is

$$
x=x_{0} \pm v_{\max } t \mp \frac{a_{\max } t^{2}}{2},
$$

which is a parabola in space-time. Moreover, the time that is needed to complete this change in direction is equal to $2 v_{\max } / a_{\max }$. This could be less time than we have at our disposal, i.e., less than $t_{q}-t_{p}$, and in this case a moving object cannot travel in that specific direction at maximal speed in order to reach its destination $x_{q}$ at time $t_{q}$.

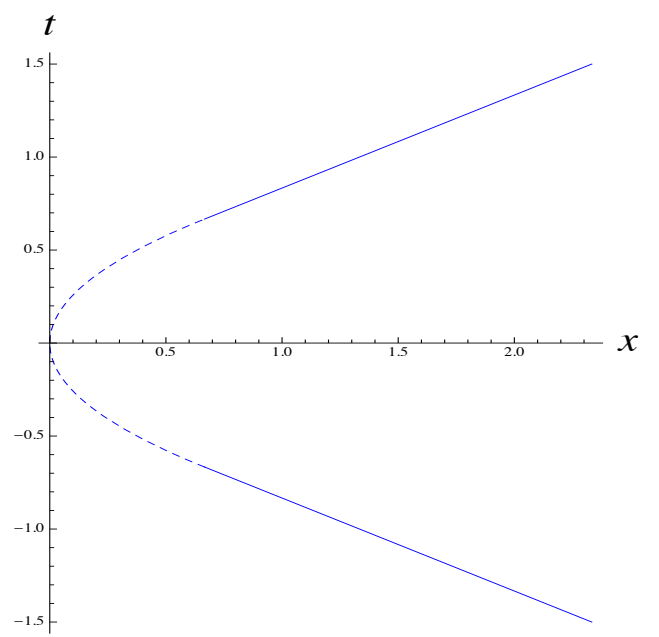

Figure 3: Changing direction on a line with constant maximal acceleration.

Figure 3 shows the space-time path of an object moving at a certain speed from right to left, this is the bottom solid line. Then the moving object slows down to a stop and accelerating again to that same speed with bounded acceleration, this is the dashed parabola. Finally, after this acceleration, the object moves from left to right again at that same certain speed, and this is the solid line at the top. Note that this is a $C^{1}$ curve.

The equation for this curve is a piecewise equation $x(t)=$

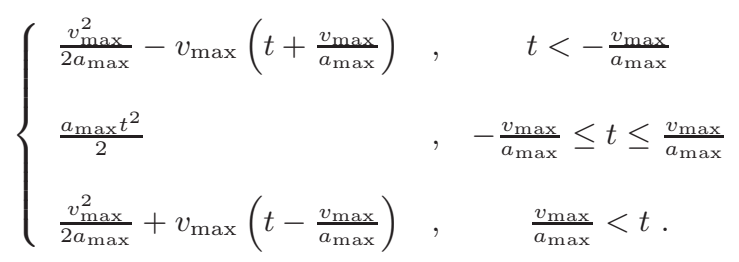

This is the most general shape of the left side of the prism. We will show next how to determine the part of this curve that bounds the kinetic prism.
First we observe that the two anchors determine a straight line of fixed slope, and that also the distance between them is fixed. This straight line can intersect the curve in Equation (1) in three different ways. It can either intersect the parabola part twice, intersect the parabola part and the straight line once each, or only intersect both straight lines. Note that we exclude the case were $\left|x_{q}-x_{p}\right| /\left(t_{q}-t_{p}\right)=v_{\max }$, as this is the case where the prism degenerates to a line and will be dealt with separately.

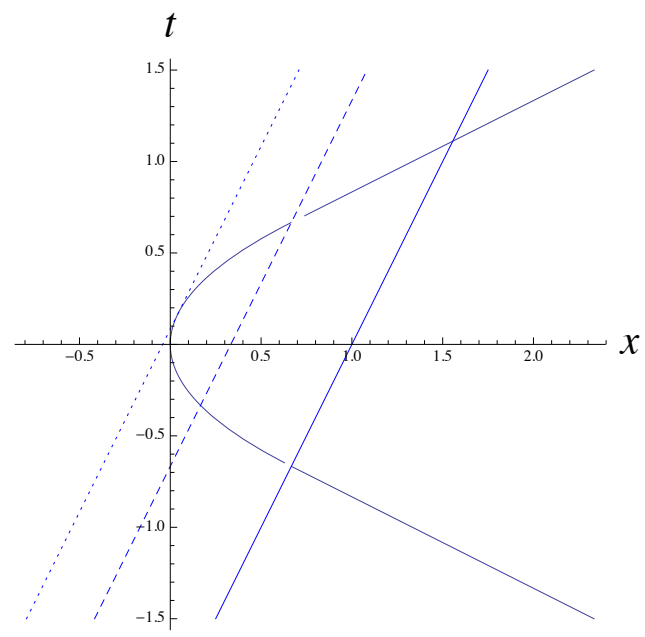

Figure 4: Delimiting the different intersection types.

Figure 4 shows how the line connecting the anchors can intersect the boundary in different ways. A line parallel to and between the dotted and the dashed line will only intersect the parabola twice. A line parallel to and between the dashed line and the solid line will intersect the parabola and the straight line once each. Finally, a line parallel to the solid line and to the right of it will intersect the straight lines once each. When $\left|x_{q}-x_{p}\right| /\left(t_{q}-t_{p}\right)=v_{\max }$, the line connecting the anchors would be parallel with one of the straight lines and only intersect the boundary curve once. Also note that if $x_{q}=x_{p}$, then the dashed and the solid line would coincide, reducing the distinct possible intersections to two and removing the case where a straight line and the parabola can be intersected.

All the lines, parallel to the line connecting the anchors, can be parametrized by the equation

$$
x_{l}=l+\frac{x_{q}-x_{p}}{t_{q}-t_{p}} t
$$

and the parameter $l$. To simplify computations we can compute the intersection points of the dashed line with the curve and denote the distance between those points by $d_{1}$, similarly we compute the intersection points of the solid line with the curve and denote the distance between those points by $d_{2}$. When we compare those distances with $d=$ $\sqrt{\left(x_{q}-x_{p}\right)^{2}+\left(t_{q}-t_{p}\right)^{2}}$ we can easily find expressions for the intersection points of the curve with the line in Equation (2), and express the distance as a function of the parameter $l$. Then we equate that expression to $d$ and solve for $l$. Finally, we use this $l$ to compute the two intersection points. 
Let $\left(x_{0}, t_{0}\right)$ be the intersection point with the smallest temporal (second) coordinate. All that remains is to translate and re-parametrize the curve in Equation (1) so that $\left(x_{0}, t_{0}\right)$ coincides with $\left(x_{p}, t_{p}\right)$. If $(x(t), t)$ is the graph of the curve in Equation (1), then

$$
\left(x_{p}-x_{0}+x\left(t-\left(t_{p}-t_{0}\right)\right), t\right) \text { where } t_{p} \leq t \leq t_{q}
$$

is the graph of the lefthand boundary of the kinetic prism between the anchors $\left(x_{p}, t_{p}\right)$ and $\left(x_{q}, t_{q}\right)$, with a speed limit $v_{\max }$ and an upper bound on acceleration $a_{\max }$.

We could use the same approach for the righthand side of the prism, but this is not necessary since the prism is point-symmetric with respect to the middle of the line segment connecting the anchors. So we merely need to apply a reflection of the lefthand boundary around this point, $\frac{\left(x_{p}, t_{p}\right)+\left(x_{q}, t_{q}\right)}{2}$, to obtain the righthand boundary.

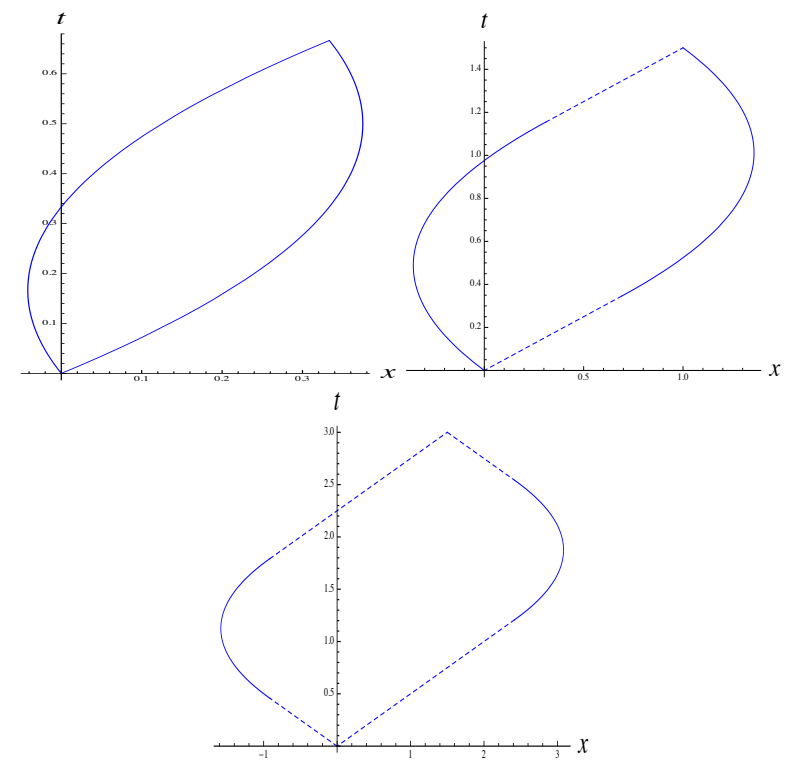

Figure 5: Three types of one-dimensional kinetic prisms.

Figure 5 shows the three distinct shapes of a one-dimensional kinetic prism. The solid part represents the change in direction at maximal acceleration of the boundary, the dashed part represents travel at maximal speed. On the top left, there is not enough time between the anchors for the object to travel at maximal speed. If it were traveling at maximal speed, the object would overshoot the anchor too early, and there would not be enough time to decelerate and change direction to make it to the second anchor in time. On the top right, the moving object could initially be traveling at maximal speed, but only between the anchors, the object cannot attain maximal speed left or right of the anchors. At the bottom of Figure 5, the object can travel at maximal speed in both directions.

Figure 6 shows a kinetic prism overlaid with a classical space-time prism. Note that in this case the sample points are relatively close together in time and that in this case, the reduction of spatio-temporal uncertainty is significant compared to the classical space-time prism. A kinetic prism

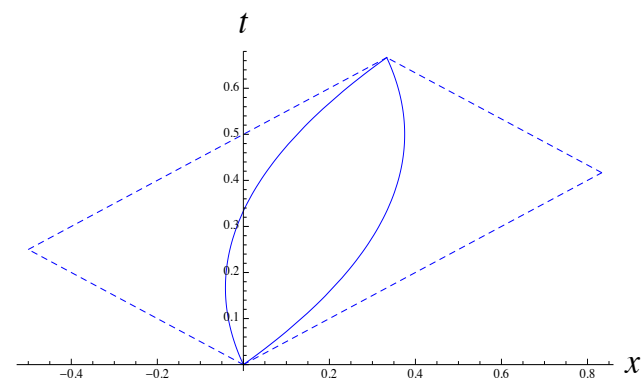

Figure 6: An overlay of a kinetic prism with a classical prism.

will resemble a classical space-time prism more as the upper bound on acceleration increases and as the temporal difference between the anchors increases.

\subsubsection{Movement in two dimensions}

For objects moving in two-dimensional space, the problem is a bit more involved. we can, however, reconstruct the boundary of the kinetic prism by reducing the problem to one-dimensional movement. If we intersect the kinetic prism with a plane that contains the line connecting the anchors, we obtain, in a sense, a subset of the trajectories restricted to this spatio-temporal plane.

Here also, we can exploit symmetry of the prism to reduce the computational cost. Note that in addition to point symmetry, the kinetic prism is also symmetric wrt to the plane which contains both anchors and is perpendicular to the spatial plane, i.e., it contains a direction parallel to the temporal axis. Taking these symmetries into account, we only need to compute a quarter of the boundary of the kinetic prism and then apply two reflections to obtain the complete boundary.

The following theorem shows that if we intersect the kinetic prism with a plane that contains both anchors, we obtain a one-dimensional prism in a sense.

Theorem 1. Let $\left(x_{p}, y_{p}, t_{p}\right),\left(x_{q}, y_{q}, t_{q}\right) \in \mathbf{R}^{2} \times \mathbf{R}$ be two anchors, where $t_{p}<t_{q}$, let $v_{\max }, a_{\max } \in \mathbf{R}^{+}$be an upper bound on speed and acceleration respectively, and let $\boldsymbol{u}=(\cos (\theta), \sin (\theta), 0)$ be any spatial direction. Let $\gamma$ be the plane spanned by the vector $\left(x_{q}-x_{p}, y_{q}-y_{p}, t_{q}-t_{p}\right)$ and the vector $\boldsymbol{u}$ and let $\beta$ be the plane spanned by the vector $\boldsymbol{u}$ and the vector $(0,0,1)$, and let both planes contain the anchor $\left(x_{p}, y_{p}, t_{p}\right)$.

Then the projection of the intersection of the kinetic prism and $\gamma$ onto $\beta$ is a one-dimensional kinetic prism with an upper bound on acceleration $a_{\max }$ and an upper bound on speed equal to

$$
\sqrt{\begin{array}{c}
\left(\cos \left(\theta_{0}+\theta\right) \frac{x_{q}-x_{p}}{t_{q}-t_{p}}+\sin \left(\theta_{0}+\theta\right) \frac{y_{q}-y_{p}}{t_{q}-t_{p}}\right)^{2} \\
-\left(\frac{x_{q}-x_{p}}{t_{q}-t_{p}}\right)^{2}-\left(\frac{y_{q}-y_{p}}{t_{q}-t_{p}}\right)^{2}+v_{\max }^{2}
\end{array}}
$$

where $\theta_{0}$ is the angle between the vectors $(1,0)$ and $\left(x_{p}-\right.$ 
$\left.x_{q}, y_{p}-y_{q}\right)$, and equal to zero if both anchors share the same location.

ProOF. We omit the proof which is based on straightforward geometric computations.

Theorem 1 is key to the algorithm to compute the boundary of the two-dimensional kinetic prism. The algorithm essentially does the same as described in Section 4.1.1, but all the computations are parametrized by the parameter $\theta$, which parametrizes a direction in the spatial plane and ranges from 0 to $2 \pi$. As shown before, we only need to let $\theta$ range from 0 to $\pi / 2$ and apply three reflections to obtain the rest of the boundary.

Geometrically, the algorithm intersects the kinetic prism with a plane that rotates around the line that connects the anchors, and computes the boundary in that plane.

The algorithm performs the following steps in which $\theta$ appears as a parameter:

1. Compute the general shape of the boundary of the kinetic prism in the plane $\gamma$.

2. Project this general shape perpendicular onto the plane $\beta$, this is the reduction to the one-dimensional case. Note that this projection does not alter the acceleration boundary, but it does alter $v_{\max }$, as described in Theorem 1. This means that the general shape of this boundary changes continuously with $\theta$.

3. As $\theta$ changes, so does the direction of the line connecting the anchors, and thus the distance between the anchors. We compute the two points on the projected boundary, such that the line segment connecting them has the same direction as the projected line that connects the anchors, and such that the the distance between the two points equals the distance between the projected anchors.

4. The point with the smallest time coordinate has sufficient information to translate the general shape of the intersected boundary accordingly. Note that the temporal distance between the intersection points on the projected boundary is the same on the actual boundary.

5. The previous steps result in a quarter of the boundary of the kinetic prism, we apply the following reflections on this boundary to obtain the other three parts.

(a) Apply a point-reflection with respect to the middle of the line segment connecting the anchors, i.e., $\left(\left(x_{0}, y_{0}, t_{0}\right)+\left(x_{1}, y_{1}, t_{1}\right)\right) / 2$.

(b) Apply a reflection with respect to the plane that contains both anchors and the direction that corresponds to $\theta=0$.

(c) Finally apply the composition of both reflections mentioned above.
Technically, it is possible to solve the equation in step 3 and obtain at most three different expressions for this solution so that we have solutions for all $\theta$. The result is a function that depends on the parameters $\theta$ and $t$, which is enough to plot the surface that bounds the kinetic prism. The software we used, Mathematica, however, will solve the equation in a discrete number of points and interpolate the result to obtain a plot. This is sufficient for our purpose.

Figure 7 shows the distinct possible shapes of the boundary between two anchors $p$ and $q$. The top left figure shows only an acceleration boundary, the top right picture shows an acceleration boundary and a maximal speed boundary both touching the anchors, and the bottom picture shows a kinetic prism where trajectories can start with maximal speed in all directions.

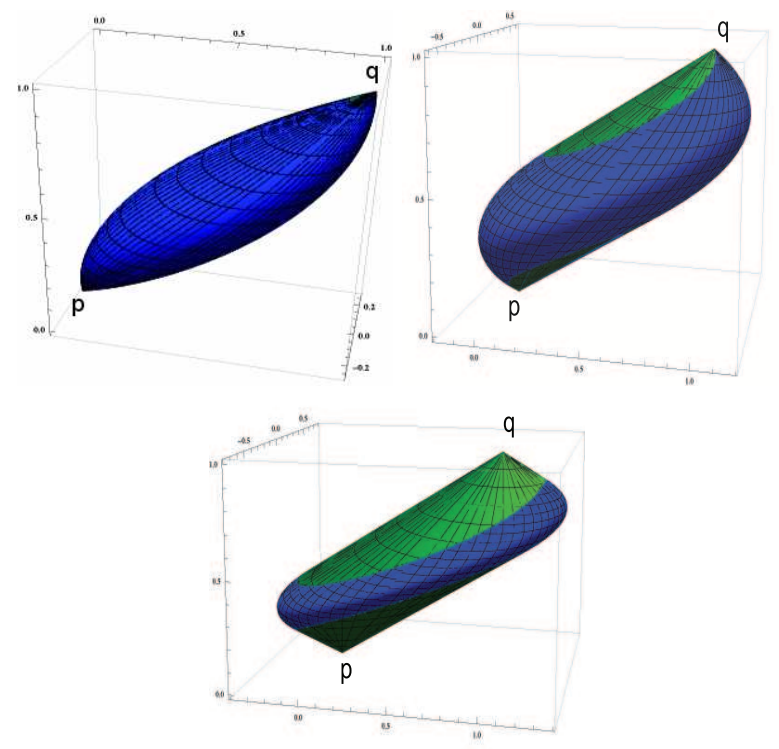

Figure 7: Three types of kinetic prisms.

\subsubsection{Analytical characterizations}

Unlike a classical space-time prism, we have only presented algorithms to compute the boundary, but not an analytical characterization of the interior of a kinetic prism. This is significantly harder for a kinetic prism and falls beyond the scope of this paper. We will, however, describe how to get to a characterization for the prisms in this section.

Note that the boundary, in general, can consist of three distinct parts, namely a boundary characterized by the maximal speed, and a boundary characterized by the maximal acceleration. Moreover, notice that the temporal width of the acceleration boundary is determined by the speed limit, i.e., the lower the speed limit, the smaller the acceleration boundary is. In fact, if the speed limit tends to $v_{\min }$, then the acceleration boundary disappears. This is the key to construct an analytical characterization of the kinetic prism.

Note that, using quantifiers, the boundary can be described in first-order logic over the reals, and, again using quantifiers, we can express membership to the boundary of a spatio-temporal point in that same logical language. Then, 
if we quantify the speed limit and let it range from $v_{\max }$ to $v_{\min }$, we effectively squeeze the boundary of the kinetic prism to a line connecting the anchors, i.e., to a degenerate prism. This characterizes the interior of the prism. The quantifiers make this characterization descriptive and not very useful. Fortunately, arithmetic over a real closed ordered field admits quantifier elimination [20,3,4], and there exists an algorithm to do just that, which, would return a quantifier free formula that would evaluate to true if the query point is part of the kinetic prism, and false otherwise.

Sometimes other ways to obtain a quantifier-free formula can be found using manual methods [10]. This is, however, food for future work.

\subsection{Initial speed and initial heading}

This case is an open problem, in the sense that we can only present how to compute the bottom cone of the kinetic prism with these constraints. We cannot apply the same approach as detailed in Section 4.1.2 because if the plane that contains both anchors does not contain the initial heading, then there is no trajectory in that plane with the given initial speed and heading. In fact, if we want to use the rotating plane approach, all the initial headings would have to have the same direction as the line connecting the anchors, and thus all anchors would have to be on the same line. This is, however, a much too unrealistic and restrictive constraint.
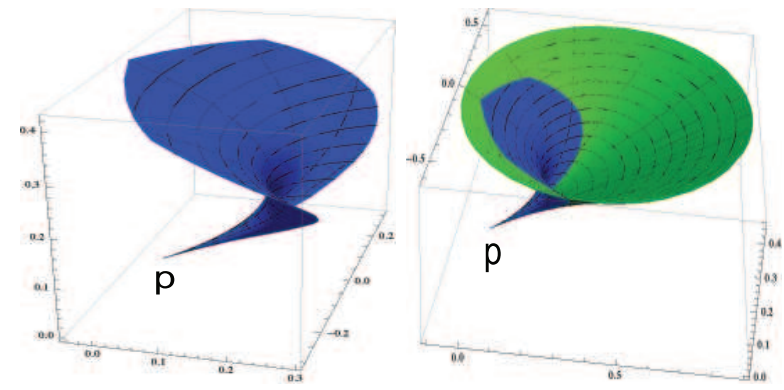

Figure 8: The acceleration and $v_{\max }$ boundary of the bottom cone.

Figure 8 shows how an object could move away from its initial heading at maximal acceleration, the boundary represents the moment when the object has reached the maximal speed and stops accelerating. The green part, the boundary that was added in the figure on the right, then represents movement at the maximal allowed speed.

We obtain a clearer view of the impact an initial heading has on the symmetry when we take the border of Figure 8 on the left. This is shown in Figure 9 on the left, and its projection on the right.

Figure 9 on the right shows the delimitation of the area a moving object can reach when moving at its constant maximal acceleration bound, given an initial speed and heading (to the right), and an initial location in the origin of the graph. This is only the bottom cone, and the only remaining symmetry is with respect to a plane that contains the initial heading and is perpendicular to the spatial plane. If this plane does not contain the second anchor, then the

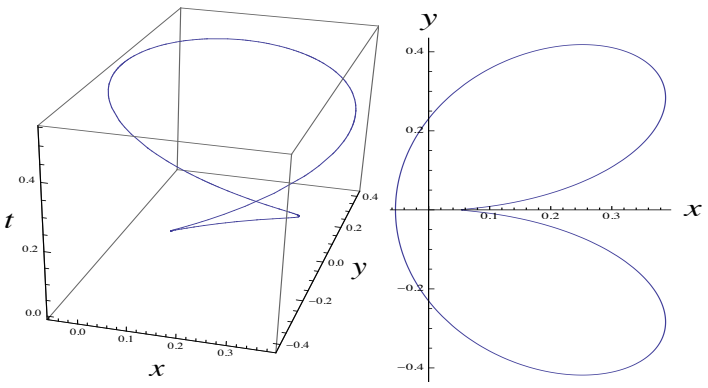

Figure 9: The acceleration boundary of the bottom cone and its projection.

symmetry we had, with respect to a plane containing both anchors and which is perpendicular to the spatial plane, is completely gone. This is partly where the difficulty lies to devise an algorithm to compute the boundary of a kinetic prism where the anchors have an initial speed and heading.

\subsection{Initial speed but no initial heading}

If there is no preferred initial heading, but only an initial speed, we can apply the rotating plane approach. Because every plane that contains both anchors will contain precisely two initial headings per anchor, and a planar trajectory from one anchor to the other that satisfies the given constraints, if they are not too strict.

The shape of the bottom cone is basically the shape of the cone described in Section 4.2, rotated around a line through the bottom anchor, parallel to the temporal axis. This has the interesting, but not surprising, effect of creating spatiotemporal bubbles inside the kinetic prism. These bubbles are volumes inside the prism that cannot be reached given the constraints on speed and acceleration.
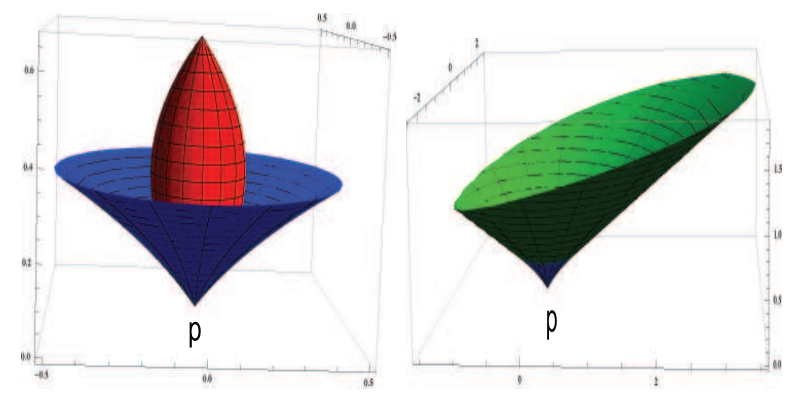

Figure 10: The acceleration and $v_{\max }$ boundary of the bottom cone.

Figure 10 on the left shows the boundary of a moving object moving with an initial speed from an anchor, given upper bounds on its speed and acceleration. The blue, funnelshaped, part contains the part of the trajectory where the object accelerates at its maximal acceleration away from the anchor and from the initial speed. The red, closed-surface within, part, similarly, contains the part of the trajectory where the object accelerates at its maximal acceleration towards the anchor and from the initial speed. This is the spatio-temporal bubble and contains the spatio-temporal points that cannot be reached. The green part, the added 
boundary in Figure 10 on the right, contains the part of the trajectories where the object has reached, and is traveling at its maximal speed.

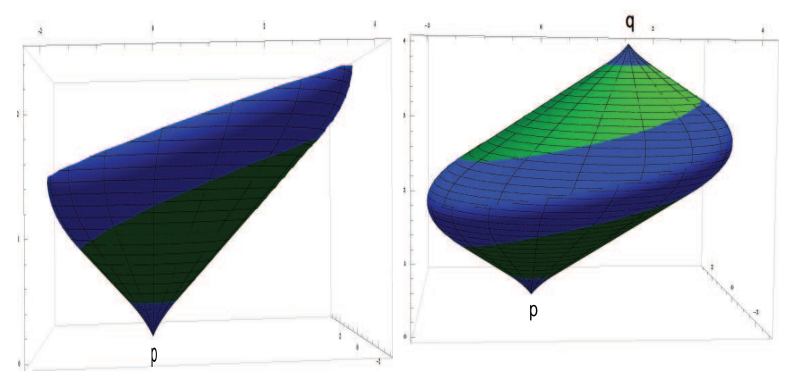

Figure 11: The full bottom cone and the the kinetic prism.

Figure 11 on the left shows the bottom part of the kinetic prism. The blue part, the extra boundary that was added to Figure 10 on the right, is the region where the object changes direction towards the second anchor at maximal acceleration in order to reach it in time. Figure 11 on the right shows the full kinetic prism.

This general structure of the kinetic prism allows for several exotic spatio-temporal topologies. The bubbles we presented behave quite nicely. They could, however, also touch, intersect each other or even the outer boundary. These more complex cases are material for future work.

\subsubsection{An initial speed range but no initial heading}

Note that there is a unique cone of directions that can be fitted between the bubble and the outward acceleration boundary, and this cone corresponds to a speed limit equal to the initial speed. This is not always the case. As the title of this section reveals, if we allow a range of initial speeds instead of one initial speed, then the bubble still touches the acceleration part in the anchor, but a cone of directions between those parts is no longer unique.

\section{CONCLUSION}

We extended the classical space-time prism by imposing an upper bound on the moving object's acceleration. In addition to this new bound on acceleration, we found that we could impose initial conditions at the anchors which have a significant impact on the kinetic prism's topology.

These different cases open new avenues for research. The open problems that appeared in this paper include the following.

- An analytical characterization of the interior of the kinetic prism is still missing.

- The two-dimensional kinetic prism is very costly to generate, this is partly due to the parametrized equation that requires solving for each parameter. A more efficient method is desirable.

- A method for different initial headings still eludes us.
- A thorough study to extend this framework to movement in higher dimensions is also desirable. Movement in three dimensions is common in ecology, namely when tracking birds. It seems, however, non-trivial to extend this to three dimensions.

- The alibi query is a boolean query that asks whether two moving objects could have met or not. This query has immediate applications in ecology. It is not clear however, how to tackle this problem.

- In addition to the alibi query, it is interesting to figure out how to quantify the sensitivity of these kinetic prisms, and their intersections, to errors on their parameters.

This list is not exhaustive, but those are the immediate challenges that arose during the research for this paper. Of these, the second item on the list needs to be tackled first in order to apply this to real-life applications.

\section{REFERENCES}

[1] K. Ahn and H. Rakha. The effects of route choice decisions on vehicle energy consumption and emissions. Transportation Research Part D: Transport and Environment, 13(3):151-167, May 2008.

[2] Kyoungho Ahn, Hesham Rakha, Antonio Trani, and Michel Van Aerde. Estimating vehicle fuel consumption and emissions based on instantaneous speed and acceleration levels. Journal of Transportation Engineering, 128(2):182-190, 2002.

[3] Saugata Basu. New results on quantifier elimination over real closed fields and applications to constraint databases. J. ACM, 46(4):537-555, 1999.

[4] Saugata Basu, Richard Pollack, and Marie-Françoise Roy. On the combinatorial and algebraic complexity of quantifier elimination. J. ACM, 43(6):1002-1045, 1996.

[5] Francesca Cagnacci, Luigi Boitani, Roger A Powell, and Mark S Boyce. Animal ecology meets gps-based radiotelemetry: a perfect storm of opportunities and challenges. Philos Trans $R$ Soc Lond B Biol Sci, 365(1550):2157-62, 2010.

[6] S.A. Cushmann. Animal movement data: Gps telemetry, autocorrelation and the need for path-level analysis. Spatial Complexity, Informatics, and Wildlife Conservation, 131, 2009.

[7] T. Hägerstrand. What about people in regional science? Papers of the Regional Science Association, 24:7-21, 1970.

[8] Jeffrey Hood, Elizabeth Sall, and Billy Charlton. A GPS-based bicycle route choice model for san francisco, california. Transportation Letters: The International Journal of Transportation Research, 3(1):63-75, 2011.

[9] Robert Knighton and Christophe Claramunt. An aeronautical temporal gis for post-flight assessment of navigation performance. T. GIS, 5(1):53-66, 2001.

[10] Bart Kuijpers, Rafael Grimson, and Walied Othman. An analytic solution to the alibi query in the space-time prisms model for moving object data. Int. J. Geogr. Inf. Sci., 25:293-322, February 2011. 
[11] Bart Kuijpers, Harvey J. Miller, Tijs Neutens, and Walied Othman. Anchor uncertainty and space-time prisms on road networks. International Journal of Geographical Information Science, 24(8):1223-1248, 2010.

[12] Bart Kuijpers and Walied Othman. Modeling uncertainty of moving objects on road networks via space-time prisms. International Journal of Geographical Information Science, 23(9):1095-1117, 2009.

[13] Bart Kuijpers and Walied Othman. Trajectory databases: Data models, uncertainty and complete query languages. J. Comput. Syst. Sci., 76(7):538-560, 2010.

[14] H.J. Miller. A measurement theory for time geography. Geographical Analysis, 37(1):17-, 2005.

[15] Barrett O'Neill. Elementary Differential Geometry. Academic Press, www.apnet.com, $2^{\text {nd }}$ edition, 1997. 482 pages.

[16] W. Othman. Implementations of spatio-temporal algorithms, 2007. http://othmanw.submanifold.be.

[17] John Parkin and Jonathon Rotheram. Design speeds and acceleration characteristics of bicycle traffic for use in planning, design and appraisal. Transport Policy, 17(5):335-341, September 2010.

[18] Dieter Pfoser and Christian S. Jensen. Capturing the uncertainty of moving-object representations. In Ralf Hartmut Güting, Dimitris Papadias, and Frederick H. Lochovsky, editors, Advances in Spatial Databases, 6th International Symposium, SSD'99, Hong Kong, China, July 20-23, 1999, Proceedings, volume 1651 of Lecture Notes in Computer Science, pages 111-132. Springer, 1999.

[19] Eva Rose, Peter Nagel, and Daniel Haag-Wackernagel. Spatio-temporal use of the urban habitat by feral pigeons (columba livia). Behavioral Ecology and Sociobiology, 60:242-254, 2006. 10.1007/s00265-006-0162-8.

[20] A. Tarski. A decision method for elementary algebra and geometry. RAND Reports, 1948.

[21] Stanley M. Tomkiewicz, Mark R. Fuller, John G. Kie, and Kirk K. Bates. Global positioning system and associated technologies in animal behaviour and ecological research. Philosophical transactions of the Royal Society of London. Series B, Biological sciences, 365(1550):2163-76, July 2010. 